Angesichts des hohen Ausmaßes an geschlechtsspezifischer Gewalt gegen Frauen und dieser Zugangsschwierigkeiten zum bestehenden Unterstützungssystem hat die Bundesregierung beschlossen, ein bundesweites Hilfetelefon „Gewalt gegen Frauen“ beim BAFzA in Köln einzurichten. Das Gesetz hierzu ist im März 2012 in Kraft getreten.

Das Hilfetelefon wird ein auf Dauer angelegtes, kostenloses telefonisches Erstberatungs- und Weitervermittlungsangebot für Frauen in allen Gewaltsituationen sein, aber auch für deren soziales Umfeld und die (Fach-)Öffentlichkeit. Unter einer einheitlichen Rufnummer wird es täglich rund um die Uhr entgeltfrei erreichbar, mehrsprachig und barrierefrei sein. Die Beratungen sind grundsätzlich anonym.

Das Hilfetelefon baut konzeptionell auf dem bestehenden Hilfesystem auf und setzt ein Netz von Unterstützungseinrichtungen vor Ort voraus. Es vervollständigt das Unterstützungssystem, indem es für betroffene Frauen die Schwelle zu Erstberatung, Unterstützung und Informationen senkt und sie ins Hilfesystem vor Ort führt. Diese Erstberatung wird durch erfahrene qualifizierte weibliche Fachkräfte erfolgen.

Nach dieser Tour durch die Geschichte jetzt zur Frage: Wie geht es weiter?

Für die Zukunft plant das BMFSFJ die Einführung eines bundesweiten Monitorings auf der Bundes- und Länderebene. Mit diesem sollen kontinuierlich und in regelmäßigen Abständen Ausmaß, Formen und Folgen von häusli- cher und sexueller Gewalt sowie die Wirkungen der AntiGewalt-Politik gemessen werden. Langfristiges Ziel ist es, eine fundierte daten- und wissensbasierte Politik im Bereich Gewalt gegen Frauen in Bund, Ländern und vor Ort zu ermöglichen.

Die Einführung eines solchen Monitorings wird auch international immer mehr eingefordert, damit Daten nicht mehr nur punktuell im Rahmen einzelner empirischer Studien erhoben werden.

Ein solches Monitoring ist nur möglich, wenn alle Stellen und Organisationen auf Bundes- und Länderebene mitmachen und ihre Daten zur Verfügung stellen bzw. neue Daten erheben. Wir würden uns freuen, wenn der djb uns bei dieser Mammutaufgabe unterstützten könnte.

Was die durch die „Bestandsaufnahme zur Situation der Frauenhäuser, Fachberatungsstellen und anderer Unterstützungsangebote für gewaltbetroffene Frauen und deren Kinder" zu Tage getretenen Schwachstellen des Hilfesystems angeht, bedarf es ebenfalls des Zusammenwirkens aller maßgeblichen Akteur/inn/e/n in Bund, Ländern und Kommunen. Es ist daher folgerichtig, dass die Gutachten Anforderungen und Empfehlungen enthalten, die in die Zuständigkeit von Bund, Ländern und Kommunen fallen.

Auch hier zählen wir weiterhin auf einen konstruktiven fachpolitischen Austausch mit dem djb!

Vielen Dank.

\title{
Zehn Jahre Gewaltschutzgesetz: Länderumfrage 2011 Erreichtes und neue alte Aufgaben
}

Susanne Köhler

Vorsitzende der Fachkommission Gewalt gegen Frauen und Kinder des djb; Rechtsanwältin, Dresden

\section{A. „Wer schlägt, muss gehen“}

Am 1. Januar 2002 trat das Gewaltschutzgesetz (GewSchG) in Kraft. Es war der in der Gesellschaft weithin sichtbare Meilenstein, der den gesellschafts- und rechtspolitisch gewollten Paradigmenwechsel markierte: Gewalt in Familie und sozialem Nahraum sollte nicht mehr länger Privatsache, die Familie nicht mehr länger ein rechtsfreier Raum sein, in den der Staat sich nicht einzumischen hat.

$\mathrm{Zu}$ diesem Zweck wurde eine Interventionskette mit ineinandergreifenden Maßnahmen aus verschiedenen Rechtsbereichen konzipiert, die orientiert an der Vorgabe „Wer schlägt, muss gehen “ nicht den erwachsenen und kindlichen Opfern dieses gesellschaftlichen Phänomens das Ausweichen abverlangte, sondern den Täter konsequent verfolgt und mit seiner Verantwortung konfrontiert.

Um den durch diese Interventionskette beabsichtigten Schutz der Opfer vor neuer Gewalt zu erreichen, bedurfte es der vernetzten Zusammenarbeit der in den verschiedenen Professionen tätigen Akteur/inn/e/n bei Polizei, Staatsanwaltschaft, Amtsgericht in Zivilsachen, Familiengericht und Opferunterstützungseinrichtungen.

Um die Prävention und den Schutz vor geschlechtsspezifischer Gewalt weiter aktiv mitgestalten zu können, war und ist es für den djb unerlässlich, sich auf eine möglichst solide Datenbasis zu stützen. Daher führte der djb bereits unmittelbar nach Inkrafttreten des Gewaltschutzgesetzes, in den ersten Wochen des Jahres 2002, eine erste bundesweite Umfrage mit dem Ziel durch, die Vorhaben der Bundesländer zur Umsetzung der neuen Gewaltschutzverfahren von Beginn an zu erfassen. Es zeigte sich seinerzeit, dass in der Bundesrepublik Deutschland noch lange nicht von einer konzertierten Aktion verschiedenster Politik- und Handlungsebenen zur Bekämpfung der Partnergewalt gesprochen werden konnte. ${ }^{1}$ 1 djb-Umfrage 2002, 〈www.djb.de/static/common/download.php/
save/45/gewaltschutzgesetz.pdf〉. 


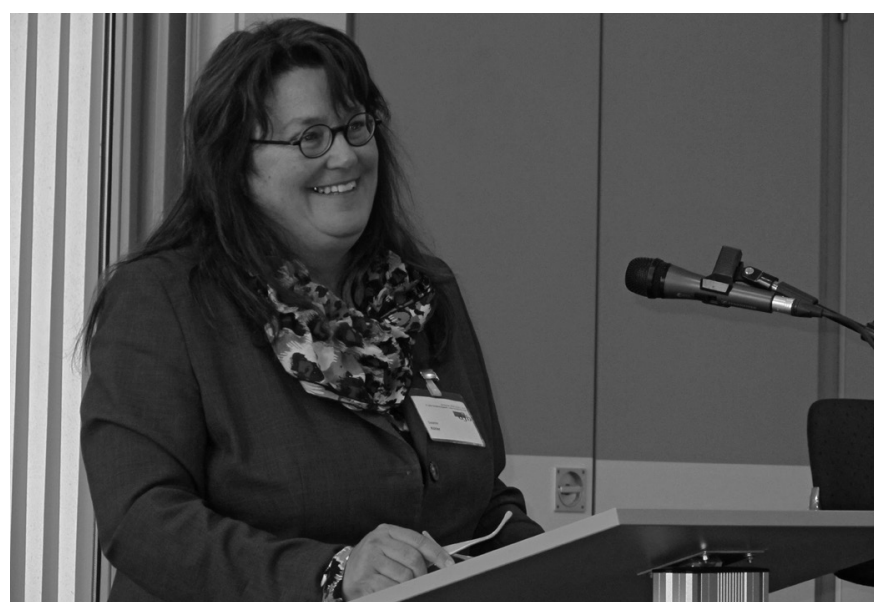

$\Delta$ Rechtsanwältin Susanne Köhler, Fachanwältin für Familienrecht, Vorsitzende der Fachkommission Gewalt gegen Frauen und Kinder, Dresden, stellte die Ergebnisse der Länderumfrage 2011 der Fachkommission Gewalt gegen Frauen und Kinder des djb dar (Foto: Rebecca Salome Freudenberg).

Diese Umfrage führte der Verband ein zweites Mal im Jahr 2004 durch. Die Ergebnisse zeigten erfreulicherweise erste Fortschritte in der Umsetzung des GewSchG. So etwa in Form von intensiverer Fortbildung der angesprochenen Professionen sowie der Verabschiedung von Landesaktionsplänen, die von Landesregierungen beschlossen worden waren, und der teils erstmaligen Gründung von Runden Tischen vor Ort. Wahrzunehmen war zudem, dass Vernetzungsgremien in Deutschland sich in ihren gemeinsamen Bemühungen um Zusammenarbeit gestärkt sahen. ${ }^{2}$

In Anbetracht der zehnjährigen Anwendung des Gewaltschutzgesetzes Anfang 2011 konzipierte der djb eine dritte bundesweite Bestandsaufnahme.

\section{B. Design der Umfrage}

Der djb hat die neue bundesweite Länderumfrage am 27. Mai 2011 gestartet. Wie schon bei den vorherigen Länderumfragen des djb wurde die Befragung an die Ressorts im Bereich Inneres, Justiz und Soziales bzw. Frauen und Gleichstellung adressiert. Von den Landesregierungen wurden die Antworten zusammengefasst übersandt. Alle 16 Bundesländer haben dankenswerterweise geantwortet. Die den Bundesländern übersandten Fragestellungen waren insgesamt in drei Bereiche aufgegliedert.

- Der Fokus im Zivilrecht orientiert sich an den Erfahrungen mit dem am 1. Januar 2002, also vor zehn Jahren, in Kraft getretenen Gewaltschutzgesetz. Aus der forensischen Praxis der Justiz sollte u.a. eruiert werden, ob und inwiefern das Beschleunigungsgebot im familiengerichtlichen Verfahren und das sogenannte große Familiengericht die gerichtliche Arbeit im Rahmen des Gewaltschutzgesetzes beeinflussen.

- Im Mittelpunkt der dem Strafrecht zuzuordnenden Fragen stehen die Spezialisierung der mit Verfahren wegen häuslicher Gewalt befassten Dezernent/inn/en, deren Arbeitsbedingungen insbesondere unter Berücksichtigung des notwendigen Pensums zur Gewährleistung der erforderlichen Vernetzungsarbeit und, perspektivisch, die Umsetzung von
„Täterarbeit“, also des Einsatzes von Verhaltenstrainings zur Vermeidung häuslicher Gewalt in konkreten Konfliktsituationen.

- Der letzte, in der Frage der Umsetzung des Paradigmenwechsels weg von häuslicher Gewalt als Privatangelegenheit hin zu einem von der Gesellschaft zu ächtenden und zu ahndenden Phänomen maßgebliche Blickwinkel umfasst Fragen zur Vernetzung der Akteur/inn/e/n im Feld der Arbeit gegen häusliche Gewalt, der Strukturierung dieser Arbeit und der Identifizierung neuer Aufgaben in diesem Bereich.

Zudem sollten nunmehr auch die Folgen zunehmender Engpässe in der Finanzierung der Beratungseinrichtungen abgefragt werden. ${ }^{3}$

\section{Wesentliche Ergebnisse der Umfrage 2011}

\section{Was ist häusliche Gewalt - Verständnis und Definition}

Bei den Antworten fällt immer wieder auf, dass der Begriff häusliche Gewalt von den Bundesländern teilweise unterschiedlich interpretiert wird. In wenigen Ländern scheint der Begriff eng ausgelegt auf die Partnerschaftsgewalt beschränkt zu sein. Die überwiegende Zahl der Bundesländer hat hingegen - möglicherweise bedingt durch die Weiterentwicklung der Arbeit - die Gewalt gegen Kinder, vereinzelt aber auch gegen andere Familienangehörige als Betroffene häuslicher Gewalt mit in den Fokus einbezogen. Eine einheitliche Definition des Begriffs der häuslichen Gewalt ist den Antworten jedenfalls nicht zu entnehmen.

Die Umfrage hat insbesondere auf die entsprechende Frage im staatsanwaltschaftlichen Bereich Folgendes ergeben:

Nicht nur bundesweit sondern auch teilweise landesweit ${ }^{4}$ werden unterschiedliche Definitionen, teilweise aber auch überhaupt keine Definition ${ }^{5}$ genannt. Als häusliche Gewalt wird sowohl Partnergewalt bearbeitet, als auch sehr weitgehend jede Gewalt gegenüber Familienangehörigen oder sogar Gewalt im Kontext von (Senioren)-Wohngemeinschaften $^{6}$, teilweise wird eine (bestehende oder frühere) häusliche Gemeinschaft verlangt, überwiegend wird dies jedoch nicht als Voraussetzung angesehen. Auch die der Einordnung als häusliche Gewalt zugrundeliegenden Deliktsformen unterscheiden sich stark: Teilweise handelt es sich nur um Körperverletzungen und ähnliche Delikte, teilweise werden aber auch Sexualdelikte und Kapitaldelikte genannt, in einer Staatsanwaltschaft ${ }^{7}$ zählen sogar Vermögensdelikte zu häuslicher Gewalt.

2 djb-Umfrage 2004, <http://www.djb.de/Kom/fK/KGgFuK/st0419-GewaltschutzG/>.

3 Fragenkatalog unter <http://www.djb.de/static/common/download.php/save/1218/st12-9a_GewaltschutzDok.pdf >.

4 Baden-Württemberg, Rheinland-Pfalz.

5 Sachsen, Sachsen-Anhalt.

6 Nordrhein-Westfalen.

7 Staatsanwaltschaft Heidelberg: Betrug. 


\section{Umsetzung des Gewaltschutzgesetzes}

\section{Zivilrechtliche Verfahren}

\section{a. Wie viele Verfahren gab es? - Prävalenz}

Ergebnisse aus den Antworten zu den Fragen A.I.1-3

Die statistische Erfassung von Gewaltschutzverfahren erfolgt in den einzelnen Bundesländern sehr unterschiedlich. Dies betrifft sowohl die Frage hinsichtlich der Häufigkeit der unterschiedlichen Verfahren und der jeweiligen Verfahrensdauer als auch die Frage nach der verfahrensrechtlichen Verbindung von Umgangs- und Sorgerechtsverfahren mit Gewaltschutzverfahren. Festzustellen ist auch, dass in einer erheblichen Anzahl von Bundesländern nach Einführung des FamFG nicht mehr nach den materiell-rechtlichen Grundlagen $(\mathbb{S} \mathbb{S} 1$ und 2 GewSchG) unterschieden wird. Es lässt sich erkennen, dass in der Gesamtschau die Anzahl der Gewaltschutzverfahren im Rahmen der zivilrechtlichen Auseinandersetzung in dem Gesamtzeitraum gestiegen ist. Teilweise kann jedoch eine Doppelzählung wegen Erfassung desselben Verfahrens sowohl wegen Kontaktverbot als auch Wohnungszuweisung nicht ausgeschlossen werden. Zur Verfahrensdauer können nur einige Bundesländer nähere Angaben machen, meist wird auf die Möglichkeit der Durchführung eines einstweiligen Anordnungsverfahrens und dessen typischerweise kurzen Verfahrensdauer hingewiesen. Von einer signifikanten Zunahme von Umgangs- bzw. Sorgerechtsverfahren nach Einführung des Gewaltschutzgesetzes berichtet kein Bundesland. Insgesamt ergibt das mitgeteilte Zahlenmaterial ein heterogenes, für Vergleichszwecke wenig geeignetes Bild.

\section{b. Verfahrensrechtliche Fragen}

Ergebnisse aus den Antworten zu den Fragen A.Il.1 und 2

Eine Notwendigkeit zur Einrichtung neuer Stellen wurde im deutlich überwiegenden Teil der Bundesländer ${ }^{8}$ nicht gesehen. In sechs Bundesländern ${ }^{9}$ wurde durch Erhöhung der Richter/ innenzahlen und/oder Umstrukturierung auf den Arbeitsanfall reagiert, in einem Bundesland ${ }^{10}$ lagen zu der Frage nach der Neueinrichtung von Stellen keine Erkenntnisse vor.

Eine statistische Erfassung von Verfahren mit getrennter Anhörung von Opfer und Täter ist in keinem Bundesland gegeben, sodass keine belastbaren konkreten Angaben gemacht werden konnten. Das BMJ teilte mit, dass die Frage nach getrennten Anhörungen in Fällen häuslicher Gewalt u.a. Gegenstand der vom BMJ geplanten Evaluierung des FamFG sein wird, es sollen „,in diese Untersuchung neben den Gewaltschutzverfahren auch die weiteren verfahrensrechtlichen Regelungen zum Schutz der Beteiligten in Fällen häuslicher Gewalt einbezogen werden. Der Forschungsauftrag wird vom BMJ/ Bundesamt für Justiz (BfJ) Ende 2011 ausgeschrieben und soll einen dreijährigen Untersuchungszeitraum umfassen.“

Von der Richterschaft, die teilweise angefragt wurde, wurde überwiegend angeführt, dass die getrennte Anhörung eher als hinderlich bezüglich der Sachverhaltsaufklärung betrachtet wird und sich verfahrensverlängernd auswirkt. Auch sei die Möglichkeit einer Absprache damit praktisch nicht mehr vorhanden.

Häufig erfolgte ein Hinweis darauf, dass ein Antrag auf getrennte Anhörung nur in den seltensten Fällen erfolgt, wobei in einem Bundesland ${ }^{11}$ die Möglichkeit der getrennten Anhörung ausdrücklich begrüßt wird.

\section{c. Materiellrechtliche Fragen}

Größere Einführungsschwierigkeiten bezüglich des Beschleunigungsgebotes und der Sicherstellung einer Teilnahme des Jugendamtes wurden aus keinem Bundesland berichtet. Durchgängig wird die Teilnahme des Jugendamtes als gewährleistet angesehen. Dabei wird auch berichtet, dass die Einführung des Beschleunigungsgebotes im Hinblick auf Gewaltschutzverfahren und damit verbundene Umgangs- und Sorgerechtsverfahren keine signifikante Änderung darstellt, da auch schon vorher diese Verfahren beschleunigt durchgeführt wurden.

Die aufgrund des FamFG entstandene Möglichkeit der selbstständigen einstweiligen Anordnung auch für Gewaltschutzverfahren wird ausdrücklich begrüßt, ebenso die abschließende Klärung der Zuständigkeit des großen Familiengerichts; Zeitverzögerungen und auch Arbeitsaufwand wegen Zuständigkeitsfragen würden so vermieden. Deutliche Unzufriedenheit wurde jedoch dahingehend geäußert, dass nunmehr die Familiengerichte auch für Streitigkeiten zuständig sind, die eher Nachbarschafts- bzw. Mietstreitigkeiten zuzuordnen wären.

\section{Durchbrechung der Gewaltspirale durch Strafverfolgung}

\section{a. Ressourcen bei der Staatsanwaltschaft}

\section{(1) Einrichtung von Sonderdezernaten bei den Staatsanwalt- schaften}

Hinsichtlich einer flächendeckenden Einrichtung von Sonderdezernaten „Häusliche Gewalt“ bei den Staatsanwaltschaften ist den Antworten der Bundesländer zu entnehmen, dass die in der früheren Umfrage (2004) schon beschriebene weitgehende Einrichtung von Sonderdezernaten zwar noch etwas vorangeschritten, aber doch immer noch nicht flächendeckend ${ }^{12}$ umgesetzt ist. Gerade große Bundesländer teilen mit, dass nur teilweise Sonderdezernate bestehen. Mancherorts wird in Beantwortung der Frage auf „Ansprechpartner“13 oder „Koordinatoren "14 mit Zuständigkeiten sowohl innerhalb der Behörde als auch für Kontakte nach außen für Fälle häusli-

8 Baden-Württemberg, Bremen, Hessen, Niedersachsen, NordrheinWestfalen, Rheinland-Pfalz, Saarland, Sachsen-Anhalt, Thüringen

9 Bayern, Berlin, Brandenburg, Hamburg, Schleswig-Holstein, Mecklenburg-Vorpommern.

10 Sachsen

11 Schleswig-Holstein.

12 Nicht flächendeckend in Baden-Württemberg, Bayern (dort teilweise alternativ „Ansprechpartner"), Nordrhein-Westfalen, Sachsen, Sachsen-Anhalt.

13 Bayern.

14 Nordrhein-Westfalen 
cher Gewalt hingewiesen. Diese können jedoch keine adäquate Alternative zur Einrichtung von Sonderdezernaten bieten. Sonderdezernate sind gerade deswegen so erfolgreich, weil im Rahmen der Spezialisierung auf das Gebiet häusliche Gewalt, auf die damit verbundenen strafrechtlichen, strafprozessualen und psychosozialen Fragestellungen ein Sachverstand gebündelt wird, welcher nur bei der praktischen Arbeit zu erwerben ist und gerade dort zur Anwendung kommen soll. Allein von Kommunikation mittels Ansprechpersonen nach innen und nach außen ist dies nicht zu erwarten.

Mehrere Länder führen in ihren Antworten explizit aus, dass bei ihnen Verfahren häuslicher Gewalt in besonders zuständigen Amtsanwaltschaften bearbeitet werden. Hiermit ist zwar eine sicherlich begrüßenswerte Spezialisierung der Dezernent/inn/en bei den Amtsanwaltschaften verbunden. Dies geht allerdings nicht selten einher mit der Bewertung von Fällen häuslicher Gewalt als minder schwere Kriminalität und birgt die Gefahr der Verfahrensbeendigung durch Einstellung mit Verweisung auf den Privatklageweg, weil den Amtsanwaltschaften allgemein Delikte von geringer Schwere mit häufiger Privatklageverweisung zugewiesen sind. Vor dem Hintergrund, dass die Verweisung auf den Privatklageweg bei häuslicher Gewalt nach dem Beschluss der Justizministerkonferenz von 1994 nicht erfolgen soll, außerdem nicht selten bei anfangs geringfügig erscheinenden Sachverhalten nach weiteren (u.a. auch staatsanwaltschaftlichen) Ermittlungen gravierende Taten aufgedeckt werden, die dann eine staatsanwaltliche Zuständigkeit begründen, sollte geprüft werden, ob eine generelle Zuweisung von Fällen häuslicher Gewalt an Amtsanwaltschaften überhaupt angemessen ist. Die gravierenden Fälle häuslicher Gewalt, die bei Ermittlungsbeginn in die staatsanwaltliche Zuständigkeit gehören, wie zum Beispiel Sexualdelikte, werden bei den Behörden mit grundsätzlich amtsanwaltlicher Zuständigkeit für häusliche Gewalt in den allgemeinen, nicht auf häusliche Gewalt spezialisierten Abteilungen bearbeitet. Für eine sachgerechte Bearbeitung nach heutigen Standards ist aber gerade auch in diesen Fällen eine Spezialisierung der sachbearbeitenden Dezernent/inn/en erforderlich. Hieraus folgt, dass eine Beschränkung der Spezialisierung allein auf die Amtsanwaltschaften nicht ausreichend sein kann.

In den Bundesländern, die Sonderdezernate bei den Staatsanwaltschaften eingerichtet haben, besteht teils die Regelung, dass in diesen Sonderdezernaten nicht nur die Vergehen im Rahmen häuslicher Gewalt wie Körperverletzung, Nötigung, Bedrohung, sondern auch Verbrechen wie Sexualdelikte und Kapitaldelikte, die sich im Kontext häuslicher Gewalt ereigneten, bearbeitet werden. ${ }^{15}$ Nachdem in der Praxis häufig sowohl körperliche als auch sexuelle Gewalt in derselben Beziehung auftreten und Fälle von tödlicher Partnergewalt nicht selten nach vorheriger körperlicher und sexueller Misshandlung auftreten, erscheint eine solche Regelung, die „allgemeine" Fälle häuslicher Gewalt, Sexualdelikte im Rahmen von (Ex-)Partnerschaften und Kapitaldelikte im Rahmen häuslicher Gewalt einem einzigen staatsanwaltlichen Sonderdezernat zuweist, als vorzugswürdig.

\section{(2) Pensum}

Bewertung und Erfassung von Fällen häuslicher Gewalt als eigene "Geschäfte" im Rahmen von PEBB§Y16; Bela-

stung der Sachbearbeiter/innen bei den Behörden

Anlässlich der Frage nach einem Jahrespensum der Dezernate häuslicher Gewalt wird teilweise darauf hingewiesen, dass PEBB $\mathbb{Y}$ Y kein gesondertes Geschäft für Ermittlungsverfahren wegen häuslicher Gewalt ausweist. ${ }^{17}$ Mehrere Länder teilen jedoch mit, dass den Dezernaten häusliche Gewalt - mit Blick auf die gegenüber einem allgemeinen Dezernat aufwändigere Bearbeitungsweise sowie die zusätzliche Belastung durch Teilnahme an Vernetzung - eine geringere Fallbelastung zugewiesen wird; teilweise wird bei der Zuweisung ein erhöhter Zuteilungsfaktor angewandt ${ }^{18}$ bzw. das Jahrespensum auf eine „geringere Fallzahl“19 bzw. auf zwei Drittel eines normalen Jahrespensums beschränkt ${ }^{20}$. Zum Teil wird auch, ohne einen Faktor anzugeben, geantwortet, dass der zusätzliche Aufwand „beim Dezernatszuschnitt berücksichtigt“ werde. ${ }^{21}$ In absoluten Zahlen werden wenige Antworten gegeben. Die Zahlen schwanken zwischen durchschnittlich 1.359 Neueingängen im Jahr pro Dezernent/in ${ }^{22}$ und $1000^{23}$.

\section{b. Polizeirecht}

Den Antworten der Länder zu Anzahl und Dauer der polizeilichen Platzverweise/ Wohnungsverweise bei häuslicher Gewalt lässt sich entnehmen, dass in den Ländern, die die Daten erheben und daher Angaben machen konnten, von diesem Instrument nach wie vor regelmäßig Gebrauch gemacht wird.

\section{III. (Mit-)Betroffene Kinder}

Aus Sicht des djb ist positiv festzustellen, dass die von häuslicher Gewalt durch eigenes (Mit-)Erleben physisch wie psychisch mit betroffenen Kinder als zu schützende Zielgruppe ebenfalls in den Fokus der Arbeit gestellt werden. Kinder sind nie nur Zeug/inn/en häuslicher Gewalt, sondern immer auch deren Opfer. Sie sehen und hören die Gewalt, sind eingebunden in die gewaltgeprägte Atmosphäre und werden in diesem Kontext oft auch selbst unmittelbar misshandelt. Sie werden bereits durch ein „nur“ mittelbares Erleben der Gewalt nach aktuellen wissenschaftlichen Erkenntnissen in ihrer Entwicklung häufig nachhaltig geschädigt und kopieren als Erwachsene die in der Kindheit erfahrenen Verhaltensmuster, sodass durch sie die häusliche Gewalt über Generationen weitergegeben wird - ein Kreislauf, den es zu durchbrechen gilt, will

15 Brandenburg, Mecklenburg-Vorpommern.

16 PEBB§Y, kurz für „Erarbeitung eines Systems der Personalbedarfsberechnung für den richterlichen, staatsanwaltlichen und Rechtspflegerdienst in der ordentlichen Gerichtsbarkeit", ist ein System zur Personalbedarfsberechnung für die deutschen Justizbehörden.

17 Bayern, Brandenburg, Hessen, Mecklenburg-Vorpommern.

18 Faktoren zwischen 1,4 (Berlin) und 1,5 (einzelne Staatsanwaltschaften in Niedersachsen, Nordrhein-Westfalen).

19 Eine ungenannte Staatsanwaltschaft in Rheinland-Pfalz.

20 Schleswig-Holstein.

21 Mecklenburg-Vorpommern.

22 Saarland.

23 Amtsanwaltsdezernat Schleswig-Holstein. 
man der Gewalt in der Gesellschaft nachhaltig entgegensteuern. Hierzu hat die Umfrage ergeben, dass in einigen Ländern durch Einbeziehung entsprechender Professionen wie Jugendamt, Schule und anderer Einrichtungen des (frühkindlichen) Kinderschutzes in die Vernetzungsarbeit bereits interventive und präventive Maßnahmen entwickelt wurden. Die Fokussierung sollte gerade in diesem Bereich möglichst flächendeckend ausgebaut werden.

\section{Täterarbeit}

Die Notwendigkeit von professioneller Täterarbeit als weiteres Instrument der Prävention im Rahmen nachhaltiger Bekämpfung häuslicher Gewalt ist heute unumstritten.

In vielen Bundesländern wurden demzufolge spezialisierte Täterprogramme eingerichtet, in denen überwiegend nach den Richtlinien der Bundesarbeitsgemeinschaft Täterarbeit Häusliche Gewalt (BAGTähG) gearbeitet wird, in anderen ${ }^{24}$ sind solche zumindest geplant. Da, wo diese Einrichtungen vorhanden sind, werden sie gut angenommen: Die Justiz weist den Programmen Täter im Rahmen von Erledigungen gemäß $\mathbb{S} 153 \mathrm{a}$ StPO oder als Bewährungsauflage zu, vereinzelt auch im Rahmen von sogenannten Täter-Opfer-Ausgleichen, die aber ohne Kontakt zwischen Täter und Opfer durchgeführt werden.

\section{Vernetzung}

Übereinstimmend wird von allen Ländern berichtet, dass Vernetzung stattfindet. Allerdings ist die Struktur der Vernetzung unterschiedlich ausgestaltet. Grundlage der Vernetzung ist in den meisten Ländern ein auf Landesebene verabschiedeter Aktionsplan. Dieser umfasst in einigen Ländern die Einrichtung einer zentralen Koordinierungsstelle häusliche Gewalt, die die Umsetzung der Ziele des Aktionsplans, insbesondere auch die Vernetzungsarbeit fördert und begleitet.

Zur Umsetzung der Vernetzung dienen zudem Runde Tische auf Landesebene, in Niedersachsen bisher ein interministerieller Arbeitskreis der beteiligten Ressorts, teils flächendeckende Arbeitskreise und Arbeitsgruppen auf der örtlichen Ebene, sowie Kooperationsvereinbarungen und Handlungsleitfäden.

Neben der Einbeziehung der klassisch mit häuslicher Gewalt befassten Professionen wie Polizei, Staatsanwaltschaft und Opferunterstützungseinrichtungen sowie Jugendämtern werden teilweise in die Vernetzung auch Gesundheitsämter sowie Ärzteschaft und weitere Gesundheitsberufe, aber auch Schulen und andere Akteur/inn/e/n aus dem Bereich der frühkindlichen Förderung und Elternunterstützung einbezogen. In einigen Bundesländern findet auch eine Verknüpfung mit Netzwerken zur Bekämpfung sexueller Gewalt statt. Die Vernetzung ist teils über Aktionspläne zentral gesteuert, teils den lokalen und regionalen Initiativen überlassen. Die auf der Landesebene organisierten und unterstützten Vernetzungen erscheinen umfassender und strukturierter. Ob eine weitergehende Standardisierung und/oder Zentralisierung der Steuerung auf übergeordneter Bundes- oder Landesebene wünschenswert ist, erscheint diskussionswürdig. Sie gewährleistet zwar ein einheitliches Vorgehen auf hohem Niveau. Zugleich droht indessen hierdurch die Gefahr einer Nivellierung, die örtliche Besonderheiten und Notwendigkeiten unberücksichtigt lässt.

\section{Schlussfolgerungen}

Die Bekämpfung des gesellschaftlichen Phänomens häusliche Gewalt ist noch lange nicht beendet. Frauen und Kinder, die von häuslicher Gewalt oder anderen Formen intrafamiliärer psychischer, physischer oder sexualisierter Gewalt betroffen sind, zeigen häufig posttraumatische Belastungsstörungen. Deswegen besteht die Gefahr, dass sie vor Gericht - vor allem in einem frühen Stadium nach der Trennung vom gewalttätigen Elternteil/Partner - nicht angemessen über die erlebte Gewalt berichten können. Sie benötigen Zeit und - gerade auch bei Gericht - einen geschützten Rahmen, der ihnen ein Sprechen über das vollständige Ausmaß der erlebten Gewalt tatsächlich ermöglicht. Dies kann nur in einer vom gewalttätigen Partner/Elternteil getrennten Anhörung geschehen.

Intrafamiliäre Gewaltbeziehungen sind zudem gekennzeichnet durch eine spezifische Dynamik von Macht und Ohnmacht, die eine echte konsensuale Einigung gleichstarker Partner unmöglich macht. Eine gemeinsame Anhörung von Täter und Opfer und ein auf die einvernehmliche Klärung hin ausgerichtetes Verfahren birgt daher die Gefahr, bestehende dysfunktionale Strukturen und Machtgefälle zu verfestigen und die bereits von Gewalt betroffene Seite erneut zu viktimisieren.

Der djb spricht sich daher dafür aus, in der praktischen Umsetzung des Gewaltschutzgesetzes und der damit in sachlichem Zusammenhang stehenden Verfahren die Möglichkeiten des FamFG verstärkt zu nutzen, um ein gewaltsensibles „setting" zu gewährleisten. Dies bedeutet u.a. die Durchfübrung getrennter Anhörungen und keinen Fokus auf Einvernehmen in Gewaltschutzverfahren zu setzen.

Die Arbeit mit von Gewalt Betroffenen erfordert ein hohes $\mathrm{Maß}$ an Kenntnissen über Strukturen und Dynamiken von Gewalt. Sie verlangt eine spezifische, opfergerechte Konstruktion der Verfahrensabläufe und eine belastbare Kooperationsbeziehung mit anderen Helfer/inn/e/n und Handelnden im Feld.

Der djb fordert daher eine fachliche und strukturelle Spezialisierung der beteiligten öffentlichen Einrichtungen (z.B. Ausbau von Sonderdezernaten bei den Staatsanwaltschaften, nicht ausschließlich bei Amtsanwaltschaften, bundesweit vorantreiben), eine adäquate Bemessung des Arbeitsaufwands für Fälle häuslicher Gewalt in den Schlüsseln für Pensen und Geschäftsverteilung und daraus folgend eine damit korrespondierende personelle Ausstattung der Gerichte und Staatsanwaltschaften.

Aufgrund der qualitativen wie quantitativen Reduktion in der Datenerfassung bezüglich Gewaltschutzsachen sowohl im Straf- wie in den Zivilverfahren geht das Gesamtbild zu den Bedarfen der Opfer in den zivil- wie strafrechtlichen Verfahren sowie der Gerichte und Beratungsstellen im Hinblick auf

24 Saarland: geplant für 2012. 
ihren Personalschlüssel verloren. Versorgungs- und Bearbeitungslücken lassen sich nicht fundiert skandalisieren, notwendige rechtspolitische Entwicklungen gestalten sich schwieriger. Auch zeigt sich Deutschland durch das Fehlen einer einheitlichen Datengrundlage aus auch international vergleichbaren Parametern in der weit über die Ländergrenzen hinausgehenden fachpolitischen Diskussion als nicht anschlussfähig.

Der djb mahnt daher in der Rechtspolitik und bei den Praktiker/inne/n an, intra- wie interdisziplinär eine verbindliche gemeinsame begriffliche Arbeitsgrundlage zu schaffen, die international anschlussfähig ist. Diese ist in alle von Justiz und Polizei geführten Statistiken zu integrieren. Zudem sind diese Statistiken dann, soweit nicht - wie in der polizeilichen Statistik - schon teilweise verwirklicht, im Hinblick auf den Bereich der häuslichen Gewalt auszudifferenzieren und damit aussagekräftiger zu gestalten.

Vernetzung auf lokaler, regionaler Landes- und Bundesebene ist ein grundlegender Baustein einer gelingenden Bekämpfung der häuslichen Gewalt in der Gesellschaft. In allen Bundesländern ist hier bereits viel geleistet worden. Einzubeziehende Professionen sind aber über den Kreis von Polizei, Staatsanwaltschaft und Opferunterstützungseinrichtungen hinaus der Kinder- und Jugendbereich, Gesundheitsämter und Ärzt/inn/e/n sowie der Schulbereich.

Für den djb ist es wichtig, überall nicht nur interventiv, sondern verstärkt auch präventiv, zum Beispiel in den Berei- chen Schule, Freizeit, Arbeits- und Wohnsituation die Problematik häuslicher Gewalt und die Notwendigkeit ihrer Bekämpfung deutlich zu machen. Hier sind bisher nur einzelne Ansätze erkennbar, eine verstärkte Einbeziehung tut not.

In einigen Ländern ist inzwischen die Bekämpfung des Gewaltverhaltens der Täter durch Angebote von speziell auf die Thematiken bei häuslicher Gewalt zugeschnittenen, standardisierten Verhaltenstrainings für Täter („Täterarbeit“) etabliert und wird zum Teil flächendeckend gefördert. Sehr hilfreich erscheint in diesem Zusammenhang die im September verabschiedete Reform der Strafprozessordnung ( $\$ 153$ a StPO) und der Änderung des StGB in Zusammenhang mit den Auflagen bei der Verwarnung mit Strafvorbehalt. Danach ist nunmehr mit den neuen Fristenregelungen und der ausdrücklichen Verankerung der Auflagen im Bereich der Einstellung und der Aussetzung der ausgesprochenen Geldstrafe zur Bewährung die Umsetzung eines Verhaltenstrainings Täterarbeit gesetzeskonform möglich und sinnvoll, wie es bereits seit längerer Zeit von Opfern wie auch Tätern häuslicher Gewalt gewünscht wurde.

Der djb hält einen flächendeckenden Ausbau dieses hochwirksamen Instruments zur Bekämpfung häuslicher Gewalt überall für unverzichtbar.

Zusammenfassung:

http://www.djb.de/static/common/download.php/save/1218/ st12-9a_GewaltschutzDok.pdf

\section{Gewaltschutz - die Rechte der Kinder}

\section{Susanne Köhler}

Vorsitzende der Fachkommission Gewalt gegen Frauen und Kinder des djb; Rechtsanwältin, Dresden

Bei häuslicher Gewalt gegen ein Elternteil sind Kinder immer (mit-)betroffen: Häufig erleiden sie selbst Gewalt oder beobachten sie. In jedem Fall hat dies schädigende Folgen auf die psychische Gesundheit und die Entwicklung des Kindes. Wie sich aus der Studie des BMFSFJ zu Gewalt gegen Frauen aus dem Jahr 2004 ergibt, berichteten 60 Prozent der befragten Frauen über die letzte gewaltbelastete Paarbeziehung, dass in dieser Paarbeziehung auch Kinder lebten, 57 Prozent der Befragten gaben an, die Kinder hätten die gewalttätigen Situationen gehört, 50 Prozent führten aus, die Kinder hätten die Gewaltsituationen auch selbst beobachtet. Nach Angaben der betroffenen Frauen wurde jedes zehnte Kind selbst körperlich angegriffen. ${ }^{1}$ Dabei hat jedes Kind das Recht auf gewaltfreie Erziehung, $\mathbb{S} 1631$ II BGB.

Um die juristischen Möglichkeiten des Eingreifens und des Schutzes für von Gewalt betroffene Kinder zu erfassen, muss man sich zunächst das zivilrechtliche Rechtssystem der elterlichen Sorge und des Umgangsrechtes vor Augen führen.
Zur elterlichen Sorge gehören zwei Hauptfelder: Die Personensorge, die die Bestimmung über den Aufenthalt, die gesundheitlichen Belange, die schulischen Belange etc. erfasst sowie die Vermögenssorge, $\mathbb{S} \mathbb{S} 1626$ ff. BGB.

Für das Umgangsrecht gilt: Umgang mit beiden Elternteilen entspricht in der Regel dem Wohl des Kindes, so $\mathbb{S} 1626$ III BGB, dabei sind die Eltern zum Umgang berechtigt und

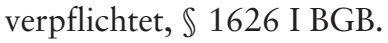

Gerichtliche Eingriffsmöglichkeiten in die elterliche Sorge erfolgen dabei auf Antrag etwa des mitsorgeberechtigten Opfers auf alleinige elterliche Sorge gemäß $\$ 1671$ BGB oder aber aufgrund einer Anregung des Jugendamtes und/oder Dritter, aber auch aufgrund von Kenntnissen des Gerichtes - so etwa im Rahmen eines Kontaktverbotsverfahrens nach $\mathbb{} 1$ Gewaltschutzgesetz - mit eigener Veranlassung gemäß $\mathbb{S} 1666$ und 1666a BGB.

Voraussetzungen für einen Eingriff gemäß $\mathbb{S} 1666$ BGB ist eine bereits eingetretene und fortwirkende oder eine gegenwärtige Gefahr des Kindeswohls, sodass sich bei weiterer

1 So BMFSFJ (Hrsg.): Lebenssituation, Sicherheit und Gesundheit von Frauen, 2004, <http://www.bmfsfi.de/BMFSFJ/Service/Publikationen/publikationen,did=120792.html $>$ (Zugriff: 15.1.2013). 